\title{
EXPLICIT CONSTRUCTION OF QUADRATIC LYAPUNOV FUNCTIONS FOR THE SMALL GAIN, POSITIVITY, CIRCLE, AND POPOV THEOREMS AND THEIR APPLICATION TO ROBUST STABILITY. PART II: DISCRETE-TIME THEORY
}

\author{
WASSIM M. HADDAD \\ Department of Mechanical and Aerospace Engineering, Florida Institute of Technology, Melbourne, FL 32901, \\ U.S.A. \\ AND \\ DENNIS S. BERNSTEIN \\ Department of Aerospace Engineering, The University of Michigan, Ann Arbor, MI 48109-2140, U.S.A.
}

\begin{abstract}
SUMMARY
In a companion paper ('Explicit construction of quadratic Lyapunov functions for the small gain, positivity, circle, and Popov theorems and their application to robust stability. Part I: Continuous-time theory'), Lyapunov functions were constructed in a unified framework to prove sufficiency in the small gain, positivity, circle, and Popov theorems. In this Part II, analogous results are developed for the discrete-time case. As in the continuous-time case, each result is based upon a suitable Riccati-like matrix equation that is used to explicitly construct a Lyapunov function that guarantees asymptotic stability of the feedback interconnection of a linear time-invariant system and a memoryless nonlinearity. Multivariable versions of the discrete-time circle and Popov criteria are obtained as extensions of known results. Each result is specialized to the linear uncertainty case and connections with robust stability for state-space systems is explored.
\end{abstract}

KEY WORDS Multivariable discrete-time Popov and circle criteria Robust stability Parameter-dependent Lyapunov functions

\section{INTRODUCTION}

In a companion paper ${ }^{1}$ Lyapunov functions were constructed in a unified framework to prove sufficiency in the small gain, positivity, circle, and Popov theorems. In this Part II, analogous results are developed for the discrete-time case. As in the continuous-time case, each result is based upon a suitable Riccati-like matrix equation that is used to explicitly construct a Lyapunov function that guarantees asymptotic stability of the feedback interconnection of a linear time-invariant system and a memoryless nonlinearity. Multivariable versions of the discrete-time circle and Popov criteria are obtained as extensions of known results.

This paper was recommended for publication by editor A. Isidori

CCC 1049-8923/94/020249-17

(C) 1994 by John Wiley \& Sons, Ltd.
Received 13 March 1992

Revised 16 September 1992 
As discussed in Reference 1, there are several reasons for seeking explicit Lyapunov function constructions of these classical results. Specifically, these constructions clarify the unity of these results while strengthening ties between the time and frequency domains for both robust analysis and synthesis. By carrying out analogous discrete-time constructions, our intention in this Part II paper is to provide similar benefits for discrete-time systems. Specifically, our aim is to provide connections between these classical results and robust stability and performance analysis for discrete-time state-space systems via quadratic fixed and parameter-dependent Lyapunov bounds. For the linear uncertainty case, we give explicit characterization of the uncertainty model and provide bounds based on single Riccati equations that can effectively be used for robust discrete-time controller synthesis. Finally, it should be noted as in the continuous-time case, the stability results of the present paper could be derived as special cases of discrete-time passivity and non-expansivity theory.

\section{PRELIMINARIES}

In this section we establish definitions and notation. Let $\mathbb{R}$ and $\mathbb{C}$ denote the real and complex numbers, let $\mathbb{N}$ denote $\{1,2,3, \ldots\}$, let ()$^{\mathrm{T}}$ denote transpose, and let $I$ denote the $n \times n$ identity matrix. Furthermore, we write $\|\cdot\|_{2}$ for Euclidean norm, $\sigma_{\max }(\cdot)$ for the maximum singular value, and $M \geqslant 0(M>0)$ to denote the fact that the Hermitian matrix $M$ is nonnegative (positive) definite. In this paper a real-rational matrix function is a matrix whose elements are rational functions with real coefficients. Furthermore, a transfer function is a real-rational matrix function each of whose elements is proper, i.e., finite at $z=\infty$. A strictly proper transfer function is a transfer function that is zero at infinity. Finally, an asymptotically stable transfer function is a transfer function each of whose poles is in the open unit disk. Let

$$
G(z) \sim\left[\begin{array}{l|l}
A & B \\
\hline C & D
\end{array}\right]
$$

denote a state-space realization of a transfer function $G(z)$, that is, $G(z)=C(z I-A)^{-1} B+D$. The notation ' $\stackrel{\min }{\sim}$, is used to denote a minimal realization.

A transfer function $G(z)$ is bounded real ${ }^{2,3}$ if (1) $G(z)$ is asymptotically stable and (2) $I-G^{*}(z) G(z)$ is nonnegative definite for $|z|>1$. Alternatively, a transfer function $G(z)$ is bounded real if and only if $G(z)$ is asymptotically stable and $\|G(z)\|_{\infty} \leqslant 1$, where $\|G(z)\|_{\infty} \triangleq \sup _{\theta \in[0,2 \pi]} \sigma_{\max }\left[G\left(\mathrm{e}^{j \theta}\right)\right]$. Furthermore, $G(z)$ is called strictly bounded real ${ }^{3}$ if (1) $G(z)$ is asymptotically stable and (2) $I-G^{*}\left(\mathrm{e}^{j \theta}\right) G\left(\mathrm{e}^{j \theta}\right)$ is positive definite for all $\theta \in[0,2 \pi]$. Finally, $G(z)$ is strongly bounded real if it is strictly bounded real and $I-D^{\mathrm{T}} D>0$, where $D \triangleq G(\infty)$.

A square transfer function $G(z)$ is called positive real if ${ }^{4-6}(1)$ all poles of $G(z)$ are in the closed unit disk, and (2) $G(z)+G^{*}(z)$ is nonnegative definite for $|z|>1$. A square transfer function $G(z)$ is called strictly positive real if ${ }^{6}(1) G(z)$ is asymptotically stable and (2) $G\left(\mathrm{e}^{j \theta}\right)+G^{*}\left(\mathrm{e}^{j \theta}\right)$ is positive definite for all $\theta \in[0,2 \pi]$. Finally, a square transfer function $G(z)$ is strongly positive real if it is strictly positive real and $D+D^{\mathrm{T}}>0$, where $D \triangleq G(\infty)$.

Finally, in this paper $G$ denotes an $l \times m$ transfer function with input $u \in \mathbb{R}^{m}$, output $y \in \mathbb{R}^{l}$, and internal state $x \in \mathbb{R}^{n}$.

\section{THE SMALL GAIN THEOREM}

In this section we construct quadratic Lyapunov functions to prove sufficiency in the small gain theorem for the interconnection of a dynamic system and a norm-bounded memoryless timevarying nonlinearity. First, however, we present the discrete-time bounded real lemma. ${ }^{2}$ 
Lemma 3.1 (Discrete-time bounded real lemma)

$$
G(z) \stackrel{\min }{\sim}\left[\begin{array}{l|l}
A & B \\
\hline C & D
\end{array}\right]
$$

is bounded real if and only if there exist real matrices $P, L$, and $W$ with $P$ positive definite such that

$$
\begin{aligned}
& P=A^{\mathrm{T}} P A+C^{\mathrm{T}} C+L^{\mathrm{T}} L, \\
& 0=B^{\mathrm{T}} P A+D^{\mathrm{T}} C+W^{\mathrm{T}} L, \\
& 0=I-D^{\mathrm{T}} D-B^{\mathrm{T}} P B-W^{\mathrm{T}} W .
\end{aligned}
$$

Proof. Sufficiency follows from algebraic manipulation of (1)-(3) while necessity follows from discrete-time spectral factorization theory. ${ }^{7}$ Alternatively, the proof also follows from the continuous-time bounded real lemma ${ }^{1}$ using the bilinear transformation $s=(z-1) /(z+1)$ which transforms $G(z)$ (for the case in which $G(z)$ is analytic at $z=-1$ ) into

$$
G_{\mathrm{c}}(s)=G\left(\begin{array}{l|l}
1+s \\
\hline 1-s
\end{array}\right) \sim\left[\begin{array}{l|l}
(I+A)^{-1}(A-I) & \sqrt{2}(I+A)^{-1} B \\
\hline \sqrt{2} C(I+A)^{-1} & D-C(I+A)^{-1} B
\end{array}\right] .
$$

Specifically, $G_{\mathrm{c}}(s)$ defines a minimal realization and is bounded real if and only if $G(z)$ is bounded real. The case in which $G(z)$ has a simple pole at $z=-1$ can be treated by an expansion of $G(z)$ that separates out this pole. For details of a similar proof see Reference 4.

Next, suppose $I-D^{\mathrm{T}} D-B^{\mathrm{T}} P B>0$, where $P$ satisfies (1)-(3). Then, since

$$
W^{\mathrm{T}} W=I-D^{\mathrm{T}} D-B^{\mathrm{T}} P B,
$$

it follows that $W^{\mathrm{T}} W$ is nonsingular. Furthermore, (2) is equivalent to

$$
W^{\mathrm{T}} L=-\left(B^{\mathrm{T}} P A+D^{\mathrm{T}} C\right) .
$$

Using (5) and noting that $W\left(W^{\mathrm{T}} W\right)^{-1} W^{\mathrm{T}}$ is an orthogonal projection as in Reference 1 , it follows from (1) that

$$
P \geqslant A^{\mathrm{T}} P A+\left(B^{\mathrm{T}} P A+D^{\mathrm{T}} C\right)^{\mathrm{T}}\left(W^{\mathrm{T}} W\right)^{-1}\left(B^{\mathrm{T}} P A+D^{\mathrm{T}} C\right)+C^{\mathrm{T}} C,
$$

or, since $\left(W^{\mathrm{T}} W\right)^{-1}=\left(I-D^{\mathrm{T}} D-B^{\mathrm{T}} P B\right)^{-1}$,

$$
P \geqslant A^{\mathrm{T}} P A+\left(B^{\mathrm{T}} P A+D^{\mathrm{T}} C\right)^{\mathrm{T}}\left(I-D^{\mathrm{T}} D-B^{\mathrm{T}} P B\right)^{-1}\left(B^{\mathrm{T}} P A+D^{\mathrm{T}} C\right)+C^{\mathrm{T}} C .
$$

Thus, in this case (1)-(3) are equivalent to the single Riccati inequality (7). The following result characterizes the bounded real property in terms of a single Riccati equation.

\section{Lemma 3.2}

Let

$$
G(z) \stackrel{\min }{\sim}\left[\begin{array}{l|l}
A & B \\
\hline C & D
\end{array}\right]
$$

Then the following statements are equivalent:

(i) $A$ is asymptotically stable and $G(z)$ is strictly bounded real;

(ii) there exist positive-definite matrices $P$ and $R$ such that

$$
\begin{gathered}
I-D^{\mathrm{T}} D-B^{\mathrm{T}} P B>0, \\
P=A^{\mathrm{T}} P A+\left(B^{\mathrm{T}} P A+D^{\mathrm{T}} C\right)^{\mathrm{T}}\left(I-D^{\mathrm{T}} D-B^{\mathrm{T}} P B\right)^{-1}\left(B^{\mathrm{T}} P A+D^{\mathrm{T}} C\right)+C^{\mathrm{T}} C+R .
\end{gathered}
$$


Proof. The proof is similar to the proof of Lemma 3.2 for the continuous-time case of Reference 1. Alternatively, the result also follows from the results of Reference 8 .

Now we prove sufficiency of the small gain theorem for the feedback interconnection of a bounded real transfer function and a norm-bounded time-varying nonlinearity. For convenience define the set

$$
\Phi_{\mathrm{br}} \triangleq\left\{\phi: \mathbb{R}^{l} \times \mathbb{N} \rightarrow \mathbb{R}^{m}:\|\phi(y, k)\|_{2} \leqslant\|y\|_{2}, y \in \mathbb{R}^{l}, k \in \mathbb{N}\right\} .
$$

\section{Theorem 3.1}

Suppose

$$
G(z) \stackrel{\min }{\sim}\left[\begin{array}{l|l}
A & B \\
\hline C & D
\end{array}\right]
$$

is strictly bounded real. Then there exist positive-definite matrices $P$ and $R$ satisfying

$$
\begin{gathered}
I-D^{\mathrm{T}} D-B^{\mathrm{T}} P B>0, \\
P=A^{\mathrm{T}} P A+\left(B^{\mathrm{T}} P A+D^{\mathrm{T}} C\right)^{\mathrm{T}}\left(I-D^{\mathrm{T}} D-B^{\mathrm{T}} P B\right)^{-1}\left(B^{\mathrm{T}} P A+D^{\mathrm{T}} C\right)+C^{\mathrm{T}} C+R .
\end{gathered}
$$

Furthermore, for all $\phi \in \Phi_{\mathrm{br}}$, the function $V(x)=x^{\mathrm{T}} P x$ is a Lyapunov function for the feedback interconnection of $G(z)$ and $\phi$. Consequently, the feedback interconnection of $G(z)$ and $\phi$ is asymptotically stable for all $\phi \in \Phi_{\mathrm{br}}$.

Proof. First note that the feedback interconnection of $G(z)$ and $\phi(\cdot, \cdot)$ corresponds to the state space representation

$$
\begin{aligned}
x(k+1) & =A x(k)+B \phi(y(k), k), \\
y(k) & =C x(k)+D \phi(y(k), k) .
\end{aligned}
$$

Since $G(z)$ is strictly bounded real it follows from Lemma 3.2 that there exist positive-definite matrices $P$ and $R$ such that (10) and (11) are satisfied. Next, we use the Lyapunov candidate $V(x)=x^{\mathrm{T}} P x$ to show that the feedback interconnection (12), (13) is asymptotically stable. The corresponding Lyapunov difference ${ }^{9}$ is given by

$$
\begin{aligned}
\Delta V(x) & =x^{\mathrm{T}}(k+1) P x(k+1)-x^{\mathrm{T}}(k) P x(k) \\
& =(A x+B \phi)^{\mathrm{T}} P(A x+B \phi)-x^{\mathrm{T}} P x \\
& =x^{\mathrm{T}}\left(A^{\mathrm{T}} P A-P\right) x+\phi^{\mathrm{T}} B^{\mathrm{T}} P A x+x^{\mathrm{T}} A^{\mathrm{T}} P B \phi+\phi^{\mathrm{T}} B^{\mathrm{T}} P B \phi,
\end{aligned}
$$

or, equivalently, using (11)

$$
\begin{aligned}
\Delta V(x)= & -x^{\mathrm{T}} R x-x^{\mathrm{T}}\left(B^{\mathrm{T}} P A+D^{\mathrm{T}} C\right)^{\mathrm{T}}\left(I-D^{\mathrm{T}} D-B^{\mathrm{T}} P B\right)^{-1}\left(B^{\mathrm{T}} P A+D^{\mathrm{T}} C\right) x \\
& -x^{\mathrm{T}} C^{\mathrm{T}} C x+\phi^{\mathrm{T}} B^{\mathrm{T}} P A x+x^{\mathrm{T}} A^{\mathrm{T}} P B \phi+\phi^{\mathrm{T}} B^{\mathrm{T}} P B \phi .
\end{aligned}
$$

Next, add and subtract $\phi^{\mathrm{T}} \phi, 2 x^{\mathrm{T}} C^{\mathrm{T}} D \phi$, and $\phi^{\mathrm{T}} D^{\mathrm{T}} D \phi$ to and from (15) so that

$$
\begin{aligned}
\Delta V(x)= & -x^{\mathrm{T}} R x-x^{\mathrm{T}}\left(B^{\mathrm{T}} P A+D^{\mathrm{T}} C\right)^{\mathrm{T}}\left(I-D^{\mathrm{T}} D-B^{\mathrm{T}} P B\right)^{-1}\left(B^{\mathrm{T}} P A+D^{\mathrm{T}} C\right) x \\
& -x^{\mathrm{T}} C^{\mathrm{T}} C x+\phi^{\mathrm{T}} B^{\mathrm{T}} P A x+x^{\mathrm{T}} A^{\mathrm{T}} P B \phi+\phi^{\mathrm{T}} B^{\mathrm{T}} P B \phi+\phi^{\mathrm{T}} \phi-\phi^{\mathrm{T}} \phi \\
& +x^{\mathrm{T}} C^{\mathrm{T}} D \phi+\phi^{\mathrm{T}} D^{\mathrm{T}} C x-x^{\mathrm{T}} C^{\mathrm{T}} D \phi-\phi^{\mathrm{T}} D^{\mathrm{T}} C x+\phi^{\mathrm{T}} D^{\mathrm{T}} D \phi-\phi^{\mathrm{T}} D^{\mathrm{T}} D \phi
\end{aligned}
$$

or, equivalently,

$$
\begin{aligned}
\Delta V(x)= & -x^{\mathrm{T}} R x-x^{\mathrm{T}}\left(B^{\mathrm{T}} P A+D^{\mathrm{T}} C\right)^{\mathrm{T}}\left(I-D^{\mathrm{T}} D-B^{\mathrm{T}} P B\right)\left(B^{\mathrm{T}} P A+D^{\mathrm{T}} C\right) x \\
& +x^{\mathrm{T}}\left(B^{\mathrm{T}} P A+D^{\mathrm{T}} C\right)^{\mathrm{T}} \phi+\phi^{\mathrm{T}}\left(B^{\mathrm{T}} P A+D^{\mathrm{T}} C\right) x-\phi^{\mathrm{T}}\left(I-D^{\mathrm{T}} D-B^{\mathrm{T}} P B\right) \phi \\
& +\phi^{\mathrm{T}} \phi-x^{\mathrm{T}} C^{\mathrm{T}} C x-\phi^{\mathrm{T}} D^{\mathrm{T}} D \phi-x^{\mathrm{T}} C^{\mathrm{T}} D \phi-\phi^{\mathrm{T}} D^{\mathrm{T}} C x .
\end{aligned}
$$


Grouping the appropriate terms in (17) yields

$$
\Delta V(x)=-x^{\mathrm{T}} R x-z^{\mathrm{T}} z+\phi^{\mathrm{T}} \phi-y^{\mathrm{T}} y,
$$

where

$$
z \triangleq\left(I-D^{\mathrm{T}} D-B^{\mathrm{T}} P B\right)^{-1 / 2}\left(B^{\mathrm{T}} P A+D^{\mathrm{T}} C\right) x-\left(I-D^{\mathrm{T}} D-B^{\mathrm{T}} P B\right)^{1 / 2} \phi .
$$

Since $R$ is positive definite and $\phi^{\mathrm{T}} \phi-y^{\mathrm{T}} y \leqslant 0$ for all $\phi \in \Phi_{\mathrm{br}}$, it follows that $\Delta V(x)$ is negative definite. Hence $V(x)$ is a Lyapunov function for the feedback interconnection of $G(z)$ and $\phi$.

Next, we specialize Theorem 3.1 to the feedback interconnection of a strongly bounded real transfer function and a linear bounded real gain. Hence consider the set $\mathcal{F}_{\text {br }}$ defined by

$$
\mathcal{F}_{\text {br }} \triangleq\left\{F: \mathbb{N} \rightarrow \mathbb{R}^{m \times l}: \sigma_{\max }(F(k)) \leqslant 1, k \in \mathbb{N}\right\} .
$$

That is, $\mathcal{F}_{\mathrm{br}}$ includes those $\phi$ in $\Phi_{\mathrm{br}}$ of the form $\phi(y, k)=F(k) y$. The following corollary of Theorem 3.1 is thus immediate.

\section{Corollary 3.1}

If

$$
G(z) \stackrel{\min }{\sim}\left[\begin{array}{l|l}
A & B \\
\hline C & D
\end{array}\right]
$$

is strongly bounded real, then the feedback interconnection of $G(z)$ and $F(\cdot)$ is asymptotically stable for all $F(\cdot) \in \mathcal{F}_{\text {br }}$.

Corollary 3.1 implies that $A+B F(\cdot)(I-D F(\cdot))^{-1} C$ is asymptotically stable in the sense that the zero solution of the discrete-time time-varying system

$$
x(k+1)=\left(A+B F(k)(I-D F(k))^{-1} C\right) x(k)
$$

is asymptotically stable. Recall from Lemma 2.1 of Reference 1 that $(I-D F(k))^{-1}$ exists for all $k \in \mathbb{N}$ since $\sigma_{\max }(D)<1$ and $\sigma_{\max }(F(k)) \leqslant 1, k \in \mathbb{N}$. This result thus implies robust stability with time-varying bounded real (but otherwise unknown) uncertainty. This can be seen by simply considering the system

$$
x(k+1)=(A+\Delta A(k)) x(k),
$$

where $\Delta A(\cdot) \in \mathcal{U}_{\mathrm{br}}$ and $\mathcal{U}_{\mathrm{br}}$ is the uncertainty set

$$
\mathcal{u}_{\mathrm{br}} \triangleq\left\{\Delta A(\cdot): \Delta A(k)=B F(k)(I-D F(k))^{-1} C, k \in \mathbb{N} \text {, where } F(\cdot) \in \mathscr{F}_{\mathrm{br}}\right\} \text {. }
$$

Then it follows from Corollary 3.1 and (19) that the zero solution to (20) is asymptotically stable for all $\Delta A \in \mathcal{U}_{\mathrm{br}}$. If we restrict our attention to constant matrices $F$, then Corollary 3.1 implies that if $G(z)$ is strongly bounded real, then $A+B F(I-D F)^{-1} C$ is asymptotically stable for all $F$ satisfying $\sigma_{\max }(F) \leqslant 1$. This case was treated in Reference 10 for robust controller analysis and synthesis with $D=0$ in $\mathcal{U}_{\mathrm{br}}$.

\section{THE POSITIVITY THEOREM}

In this section we construct quadratic Lyapunov functions to prove the positivity theorem for the system interconnection considered in Section 3. Furthermore, we specialize the results to 
the case of linear uncertainty and draw connections with robust stability for positive real uncertainty.

Lemma 4.1 (Discrete-time positive real lemma) ${ }^{4,6}$

$$
G(z) \stackrel{\min }{\sim}\left[\begin{array}{l|l}
A & B \\
\hline C & D
\end{array}\right]
$$

is positive real if and only there exist real matrices $P, L$, and $W$ with $P$ positive definite such that

$$
\begin{aligned}
& P=A^{\mathrm{T}} P A+L^{\mathrm{T}} L, \\
& 0=B^{\mathrm{T}} P A-C+W^{\mathrm{T}} L, \\
& 0=D+D^{\mathrm{T}}-B^{\mathrm{T}} P B-W^{\mathrm{T}} W .
\end{aligned}
$$

Proof. Sufficiency follows from algebraic manipulation of (21)-(23) while necessity follows from discrete spectral factorization theory. Alternatively, the proof also follows from the continuous-time positive real lemma ${ }^{1}$ using the bilinear transformation $s=(z-1) /(z+1)$. For details see Reference 4.

Suppose that $D+D^{\mathrm{T}}-B^{\mathrm{T}} P B>0$ where $P$ satisfies (21)-(23). Then, since

$$
W^{\mathrm{T}} W=D+D^{\mathrm{T}}-B^{\mathrm{T}} P B,
$$

it follows that $W^{\mathrm{T}} W$ is nonsingular, and (22) implies

$$
W^{\mathrm{T}} L=-\left(B^{\mathrm{T}} P A-C\right) \text {. }
$$

Using (25) and noting as in Reference 1 that $L^{\mathrm{T}} L \geqslant L^{\mathrm{T}} W\left(W^{\mathrm{T}} W\right)^{-1} W^{\mathrm{T}} L$, it follows from (21) that

$$
\begin{gathered}
P \geqslant A^{\mathrm{T}} P A+\left(B^{\mathrm{T}} P A-C\right)^{\mathrm{T}}\left(W^{\mathrm{T}} W\right)^{-1}\left(B^{\mathrm{T}} P A-C\right), \\
\text { or, since }\left(W^{\mathrm{T}} W\right)^{-1}=\left(D+D^{\mathrm{T}}-B^{\mathrm{T}} P B\right)^{-1}, \\
P \geqslant A^{\mathrm{T}} P A+\left(B^{\mathrm{T}} P A-C\right)^{\mathrm{T}}\left(D+D^{\mathrm{T}}-B^{\mathrm{T}} P B\right)^{-1}\left(B^{\mathrm{T}} P A-C\right) .
\end{gathered}
$$

Using the Riccati inequality version of (27) to characterize positive realness, we have the following result.

\section{Lemma 4.2}

Let

$$
G(z) \stackrel{\min }{\sim}\left[\begin{array}{l|l}
A & B \\
\hline C & D
\end{array}\right] .
$$

Then the following statements are equivalent:

(i) $A$ is asymptotically stable and $G(z)$ is strongly positive real; 
(ii) there exist positive-definite matrices $P$ and $R$ such that

$$
\begin{gathered}
D+D^{\mathrm{T}}-B^{\mathrm{T}} P B>0, \\
P=A^{\mathrm{T}} P A+\left(B^{\mathrm{T}} P A-C\right)^{\mathrm{T}}\left(D+D^{\mathrm{T}}-B^{\mathrm{T}} P B\right)^{-1}\left(B^{\mathrm{T}} P A-C\right)+R .
\end{gathered}
$$

Proof. The proof is similar to the proof of Lemma 4.2 for the continuous-time case of Reference 1.

We now prove the positivity theorem for the negative feedback interconnection given a strongly positive real transfer function and a memoryless time-varying nonlinearity. For the statement of the next result we define the set

$$
\Phi_{\mathrm{pr}} \triangleq\left\{\phi: \mathbb{R}^{m} \times \mathbb{N} \rightarrow \mathbb{R}^{m}: \phi^{\mathrm{T}}(y, k) y \geqslant 0, \quad y \in \mathbb{R}^{m}, \quad k \in \mathbb{N}\right\} .
$$

\section{Theorem 4.1}

Suppose

$$
G(z) \stackrel{\min }{\sim}\left[\begin{array}{l|l}
A & B \\
\hline C & D
\end{array}\right]
$$

is strongly positive real. Then there exist positive-definite matrices $P$ and $R$ satisfying

$$
\begin{gathered}
D+D^{\mathrm{T}}-B^{\mathrm{T}} P B>0, \\
P=A^{\mathrm{T}} P A+\left(B^{\mathrm{T}} P A-C\right)^{\mathrm{T}}\left(D+D^{\mathrm{T}}-B^{\mathrm{T}} P B\right)^{-1}\left(B^{\mathrm{T}} P A-C\right)+R .
\end{gathered}
$$

Furthermore, for all $\phi \in \Phi_{\mathrm{pr}}$, the function $V(x)=x^{\mathrm{T}} P x$ is a Lyapunov function for the negative feedback interconnection of $G(z)$ and $\phi$. Consequently, the negative feedback interconnection of $G(z)$ and $\phi$ is asymptotically stable for all $\phi \in \Phi_{\mathrm{pr}}$.

Proof. First note that the negative feedback interconnection of $G(z)$ and $\phi(\cdot, \cdot)$ has the state-space representation

$$
\begin{aligned}
x(k+1) & =A x(k)-B \phi(y(k), k), \\
y(k) & =C x(k)-D \phi(y(k), k) .
\end{aligned}
$$

Since $G(z)$ is strongly positive real it follows from Lemma 4.2 that there exist positive-definite matrices $P$ and $R$ such that (30) and (31) are satisfied. Next, we use the Lyapunov candidate $V(x)=x^{\mathrm{T}} P x$ to show that the negative feedback interconnection (32), (33) is asymptotically stable. The corresponding Lyapunov difference is given by

$$
\Delta V(x)=x^{\mathrm{T}}\left(A^{\mathrm{T}} P A-P\right) x-x^{\mathrm{T}} A^{\mathrm{T}} P B \phi-\phi^{\mathrm{T}} B^{\mathrm{T}} P A x+\phi^{\mathrm{T}} B^{\mathrm{T}} P B \phi,
$$

or, equivalently, using (31)

$$
\begin{aligned}
\Delta V(x)= & -x^{\mathrm{T}} R x-x^{\mathrm{T}}\left(B^{\mathrm{T}} P A-C\right)^{\mathrm{T}}\left(D+D^{\mathrm{T}}-B^{\mathrm{T}} P B\right)^{-1}\left(B^{\mathrm{T}} P A-C\right) x \\
& -x^{\mathrm{T}} A^{\mathrm{T}} P B \phi-\phi^{\mathrm{T}} B^{\mathrm{T}} P A x+\phi^{\mathrm{T}} B^{\mathrm{T}} P B \phi .
\end{aligned}
$$

Now add and subtract $2 \phi^{\mathrm{T}} C x$ and $2 \phi^{\mathrm{T}} D \phi$ to and from (35) so that

$$
\begin{aligned}
\Delta V(x)= & -x^{\mathrm{T}} R x-x^{\mathrm{T}}\left(B^{\mathrm{T}} P A-C\right)^{\mathrm{T}}\left(D+D^{\mathrm{T}}-B^{\mathrm{T}} P B\right)^{-1}\left(B^{\mathrm{T}} P A-C\right) x \\
& -x^{\mathrm{T}} A^{\mathrm{T}} P B \phi-\phi^{\mathrm{T}} B^{\mathrm{T}} P A x+\phi^{\mathrm{T}} B^{\mathrm{T}} P B \phi+\phi^{\mathrm{T}} C x+x^{\mathrm{T}} C^{\mathrm{T}} \phi \\
& -\phi^{\mathrm{T}} C x-x^{\mathrm{T}} C^{\mathrm{T}} \phi+\phi^{\mathrm{T}} D \phi+\phi^{\mathrm{T}} D^{\mathrm{T}} \phi-\phi^{\mathrm{T}} D \phi-\phi^{\mathrm{T}} D^{\mathrm{T}} \phi
\end{aligned}
$$


or, equivalently,

$$
\begin{aligned}
\Delta V(x)= & -x^{\mathrm{T}} R x-x^{\mathrm{T}}\left(B^{\mathrm{T}} P A-C\right)^{\mathrm{T}}\left(D+D^{\mathrm{T}}-B^{\mathrm{T}} P B\right)^{-1}\left(B^{\mathrm{T}} P A-C\right) x-x^{\mathrm{T}}\left(B^{\mathrm{T}} P A-C\right)^{\mathrm{T}} \phi \\
& -\phi^{\mathrm{T}}\left(B^{\mathrm{T}} P A-C\right) x-\phi^{\mathrm{T}}\left(D+D^{\mathrm{T}}-B^{\mathrm{T}} P B\right) \phi-2 \phi^{\mathrm{T}}(C x-D \phi) .
\end{aligned}
$$

Grouping the appropriate terms in (37) yields

$$
\Delta V(x)=-x^{\mathrm{T}} R x-z^{\mathrm{T}} z-2 \phi^{\mathrm{T}} y,
$$

where

$$
z \triangleq-\left(D+D^{\mathrm{T}}-B^{\mathrm{T}} P B\right)^{-1 / 2}\left(B^{\mathrm{T}} P A-C\right) x-\left(D+D^{\mathrm{T}}-B^{\mathrm{T}} P B\right)^{1 / 2} \phi .
$$

Since $R$ is positive definite and $\phi^{\mathrm{T}}(y, k) y \geqslant 0$ for all $\phi \in \Phi_{\mathrm{pr}}$, it follows that $\Delta V(x)$ is negative definite. Hence $V(x)$ is a Lyapunov function for the feedback interconnection of $G(z)$ and $\phi$.

Next, we specialize Theorem 4.1 to the feedback interconnection of a strongly positive real transfer function and a linear gain $F(k)$ satisfying $F(k)+F^{\mathrm{T}}(k) \geqslant 0, k \in \mathbb{N}$. Hence define

$$
\mathcal{F}_{\mathrm{pr}} \triangleq\left\{F: \mathbb{N} \rightarrow \mathbb{R}^{m \times m}: F(k)+F^{\mathrm{T}}(k) \geqslant 0, k \in \mathbb{N}\right\} .
$$

\section{Corollary 4.1}

If

$$
G(z) \stackrel{\min }{\sim}\left[\begin{array}{l|l}
A & B \\
\hline C & D
\end{array}\right]
$$

is strongly positive real, then the negative feedback interconnection of $G(z)$ and $F(\cdot)$ is asymptotically stable for all $F(\cdot) \in \mathcal{F}_{\mathrm{pr}}$.

As in the bounded real case, Corollary 4.1 guarantees robust stability for the system

$$
x(k+1)=(A+\Delta A(k)) x(k),
$$

where $\Delta A(\cdot) \in \mathcal{U}_{\mathrm{pr}}$ and $\mathcal{U}_{\mathrm{pr}}$ is the uncertainty set characterized by

$$
\mathcal{U}_{\mathrm{pr}} \triangleq\left\{\Delta A(\cdot): \Delta A(k)=-B F(k)(I+D F(k))^{-1} C, k \in \mathbb{N} \text {, where } F(\cdot) \in \mathcal{F}_{\mathrm{pr}}\right\} .
$$

Recall from Lemma 2.2 of Reference 1 that $(I+D F(k))^{-1}$ exists, $k \in \mathbb{N}$, since $D+D^{\mathrm{T}}>0$ and $F(k)+F^{\mathrm{T}}(k) \geqslant 0, k \in \mathbb{N}$. Finally, if we further restrict our attention to constant matrices $F$, then Corollary 4.1 implies that if $G(z)$ is strongly positive real, then $A-B F(I+D F)^{-1} C$ is asymptotically stable for all $F$ satisfying $F+F^{\mathrm{T}} \geqslant 0$.

\section{THE CIRCLE CRITERION}

In this section we construct quadratic Lyapunov functions to prove a multivariable generalization of the discrete-time circle criterion. ${ }^{11-14}$ As in the continuous-time case most of the available results on the discrete-time circle criterion are confined to scalar strictly proper systems with a single-loop nonlinearity. Furthermore, most of the available proofs of the discrete-time circle criterion are based upon input-output properties and function-analytic methods ${ }^{13}$ or graphical techniques. ${ }^{11,12,14}$ A notable exception is Reference 15 which provides gain and phase margins for strictly proper discrete-time systems using quadratic Lyapunov functions. However, the nonlinearities considered in Reference 15 are confined to scalar sector 
boundaries $k_{1}, k_{2}$. We remove these limitations and address the multivariable case for proper systems. Application of this result to robust stability with respect to sector-bounded timevarying uncertainty is also discussed. To begin, we define the set $\Phi_{c}$ of sector-bounded timevarying memoryless nonlinearities. Let $K_{1}, K_{2} \in \mathbb{R}^{m \times l}$ be given matrices and define

$$
\Phi_{\mathrm{c}} \triangleq\left\{\phi: \mathbb{R}^{l} \times \mathbb{N} \rightarrow \mathbb{R}^{m}:\left[\phi(y, k)-K_{1} y\right]^{\mathrm{T}}\left[\phi(y, k)-K_{2} y\right] \leqslant 0, y \in \mathbb{R}^{m}, k \in \mathbb{N}\right\} .
$$

Theorem 5.1

Suppose $\left[I+K_{2} G(z)\right]\left[I+K_{1} G(z)\right]^{-1}$ is strongly positive real, where

$$
G(z) \stackrel{\min }{\sim}\left[\begin{array}{l|l}
A & B \\
\hline C & D
\end{array}\right] .
$$

Then there exist positive-definite matrices $P$ and $R$ satisfying

$$
\begin{aligned}
2 I+ & \left(K_{2}-K_{1}\right)\left(I+D K_{1}\right)^{-1} D+D^{\mathrm{T}}\left(I+D K_{1}\right)^{-\mathrm{T}}\left(K_{2}-K_{1}\right)^{\mathrm{T}} \\
& \quad-\left(I+K_{1} D\right)^{-\mathrm{T}} B^{\mathrm{T}} P B\left(I+K_{1} D\right)^{-1}>0, \\
P= & {\left[A-B\left(I+K_{1} D\right)^{-1} K_{1} C\right]^{\mathrm{T}} P\left[A-B\left(I+K_{1} D\right)^{-1} K_{1} C\right] } \\
& +\left[\left(K_{2}-K_{1}\right)\left(I+D K_{1}\right)^{-1} C-\left(I+K_{1} D\right)^{-\mathrm{T}} B^{\mathrm{T}} P\left(A-B\left(I+K_{1} D\right)^{-1} K_{1} C\right)\right]^{\mathrm{T}} \\
& \times\left[2 I+\left(K_{2}-K_{1}\right)\left(I+D K_{1}\right)^{-1} D+D^{\mathrm{T}}\left(I+D K_{1}\right)^{-\mathrm{T}}\left(K_{2}-K_{1}\right)^{\mathrm{T}}\right. \\
& \left.-\left(I+K_{1} D\right)^{-\mathrm{T}} B^{\mathrm{T}} P B\left(I+K_{1} D\right)^{-1}\right]^{-1} \\
& \times\left[\left(K_{2}-K_{1}\right)\left(I+D K_{1}\right)^{-1} C-\left(I+K_{1} D\right)^{-\mathrm{T}} B^{\mathrm{T}} P\left(A-B\left(I+K_{1} D\right)^{-1} K_{1} C\right]+R .\right.
\end{aligned}
$$

Furthermore, for all $\phi \in \Phi_{\mathrm{c}}$, the function $V(x)=x^{\mathrm{T}} P x$ is a Lyapunov function for the negative feedback interconnection of $G(z)$ and $\phi$. Consequently, the negative feedback interconnection of $G(z)$ and $\phi$ is asymptotically stable for all $\phi \in \Phi_{\mathrm{c}}$.

Proof. First note that the negative feedback interconnection of $G(z)$ and $\phi(\cdot, \cdot)$ has the state-space representation (32) and (33). Furthermore (see Reference 1), $\left[I+K_{2} G(z)\right]$ $\left[I+K_{1} G(z)\right]^{-1}$ has a minimal realization given by

$$
\left[\begin{array}{c|c}
A-B\left(I+K_{1} D\right)^{-1} K_{1} C & B\left(I+K_{1} D\right)^{-1} \\
\hline\left(K_{2}-K_{1}\right)\left(I+D K_{1}\right)^{-1} C & I+\left(K_{2}-K_{1}\right)\left(I+D K_{1}\right)^{-1} D
\end{array}\right] .
$$

Now it follows from Lemma 4.2 that since $\left[I+K_{2} G(z)\right]\left[I+K_{1} G(z)\right]^{-1}$ is strongly positive real there exist positive-definite matrices $P$ and $R$ such that (38) and (39) are satisfied. Next define the Lyapunov candidate $V(x)=x^{\mathrm{T}} P x$ and let $\phi \in \Phi_{\mathrm{c}}$. Then we obtain

$$
\Delta V(x)=x^{\mathrm{T}}\left(A^{\mathrm{T}} P A-P\right) x-x^{\mathrm{T}} A^{\mathrm{T}} P B \phi-\phi^{\mathrm{T}} B^{\mathrm{T}} P A x+\phi^{\mathrm{T}} B^{\mathrm{T}} P B \phi,
$$

or, equivalently, using (38)

where

$$
\begin{aligned}
\Delta V(x)= & -x^{\mathrm{T}} R x-x^{\mathrm{T}} Q x-x^{\mathrm{T}} C^{\mathrm{T}} K_{1}^{\mathrm{T}}\left(I+K_{1} D\right)^{-\mathrm{T}} B^{\mathrm{T}} P B\left(I+K_{1} D\right)^{-1} K_{1} C x \\
& +x^{\mathrm{T}} C^{\mathrm{T}} K_{1}^{\mathrm{T}}\left(I+K_{1} D\right)^{-\mathrm{T}} B^{\mathrm{T}} P A x+x^{\mathrm{T}} A^{\mathrm{T}} P B\left(I+K_{1} D\right)^{-1} K_{1} C x \\
& -\phi^{\mathrm{T}} B^{\mathrm{T}} P A x-x^{\mathrm{T}} A^{\mathrm{T}} P B \phi+\phi^{\mathrm{T}} B^{\mathrm{T}} P B \phi,
\end{aligned}
$$

$$
\begin{aligned}
Q \triangleq & {\left[\left(K_{2}-K_{1}\right)\left(I+D K_{1}\right)^{-1} C-\left(I+K_{1} D\right)^{-\mathrm{T}} B^{\mathrm{T}} P\left(A-B\left(I+K_{1} D\right)^{-1} K_{1} C\right)\right]^{\mathrm{T}} } \\
& \times\left[2 I+\left(K_{2}-K_{1}\right)\left(I+D K_{1}\right)^{-1} D+D^{\mathrm{T}}\left(I+D K_{1}\right)^{-\mathrm{T}}\left(K_{2}-K_{1}\right)^{\mathrm{T}}\right. \\
& \left.-\left(I+K_{1} D\right)^{-\mathrm{T}} B^{\mathrm{T}} P B\left(I+K_{1} D\right)^{-1}\right]^{-1} \\
& \times\left[\left(K_{2}-K_{1}\right)\left(I+D K_{1}\right)^{-1} C-\left(I+K_{1} D\right)^{-\mathrm{T}} B^{\mathrm{T}} P\left(A-B\left(I+K_{1} D\right)^{-1} K_{1} C\right)\right]
\end{aligned}
$$


Next, add and subtract

$$
\begin{aligned}
& 2\left[\left(I+K_{1} D\right) \phi-K_{1} C x\right]^{\mathrm{T}}\left[\left(I+K_{1} D\right) \phi-K_{1} C x\right], \\
& 2\left[\left(I+K_{1} D\right) \phi-K_{1} C x\right]^{\mathrm{T}}\left(K_{2}-K_{1}\right)\left(I+D K_{1}\right)^{-1} C x, \\
& 2\left[\left(I+K_{1} D\right) \phi-K_{1} C x\right]^{\mathrm{T}}\left(K_{2}-K_{1}\right)\left(I+D K_{1}\right)^{-1} D\left[\left(I+K_{1} D\right) \phi-K_{1} C x\right], \\
& 2\left[\left(I+K_{1} D\right) \phi-K_{1} C x\right]^{\mathrm{T}}\left(I+K_{1} D\right)^{-\mathrm{T}} B^{\mathrm{T}} P B\left(I+K_{1} D\right)^{-1} K_{1} C X
\end{aligned}
$$

to and from (41) so that (after some algebraic manipulation)

$$
\begin{aligned}
\Delta V(x)= & -x^{\mathrm{T}} R x-x^{\mathrm{T}} Q x+\left[\left(K_{2}-K_{1}\right)\left(I+D K_{1}\right)^{-1} C\right. \\
& \left.-\left(I+K_{1} D\right)^{-\mathrm{T}} B^{\mathrm{T}} P\left(A-B\left(I+K_{1} D\right)^{-1} K_{1} C\right)\right]^{\mathrm{T}}\left[\left(I+K_{1} D\right) \phi-K_{1} C x\right] \\
& +\left[\left(I+K_{1} D\right) \phi-K_{1} C x\right]^{\mathrm{T}}\left(K_{2}-K_{1}\right)\left(I+D K_{1}\right)^{-1} C \\
& \left.-\left(I+K D_{1}\right)^{-\mathrm{T}} B^{\mathrm{T}} P\left(A-B\left(I+K_{1} D\right)^{-1} K_{1} C\right)\right] \\
& -\left[\left(I+K_{1} D\right) \phi-K_{1} C x\right]^{\mathrm{T}}\left[2 I+\left(K_{2}+K_{1}\right)\left(I+D K_{1}\right)^{-1} D+D^{\mathrm{T}}\left(I+D K_{1}\right)^{-\mathrm{T}}\left(K_{2}-K_{1}\right)^{\mathrm{T}}\right. \\
& \left.-\left(I+K_{1} D\right)^{-\mathrm{T}} B^{\mathrm{T}} P B\left(I+K_{1} D\right)^{-1}\right]\left[\left(I+K_{1} D\right) \phi-K_{1} C x\right] \\
& +2\left[\left(I+K_{1} D\right) \phi-K_{1} C x\right]^{\mathrm{T}}\left[\left(I+K_{2} D\right) \phi-K_{1} C x\right] .
\end{aligned}
$$

Grouping the appropriate terms in (42) yields

$$
\Delta V(x)=-x^{\mathrm{T}} R x-z^{\mathrm{T}} z+2\left(\phi-K_{1} y\right)^{\mathrm{T}}\left(\phi-K_{2} y\right),
$$

where

$$
\begin{aligned}
z \triangleq & {\left[2 I+\left(K_{2}-K_{1}\right)\left(I+D K_{1}\right)^{-1} D+D^{\mathrm{T}}\left(I+D K_{1}\right)^{-\mathrm{T}}\left(K_{2}-K_{1}\right)^{\mathrm{T}}\right.} \\
& \left.-\left(I+K_{1} D\right)^{-\mathrm{T}} B^{\mathrm{T}} P B\left(I+K_{1} D\right)^{-1}\right]^{-1 / 2} \\
& \times\left[\left(K_{2}-K_{1}\right)\left(I+D K_{1}\right)^{-1} C-\left(I+K_{1} D\right)^{-\mathrm{T}} B^{\mathrm{T}} P\left(A-B\left(I+K_{1} D\right)^{-1} K_{1} C\right] x\right. \\
& -\left[2 I+\left(K_{2}-K_{1}\right)\left(I+D K_{1}\right)^{-1} D+D^{\mathrm{T}}\left(I+D K_{1}\right)^{-\mathrm{T}}\left(K_{2}-K_{1}\right)^{\mathrm{T}}\right. \\
& -\left(I+K_{1} D\right)^{-\mathrm{T}} B^{\mathrm{T}} P B\left(I+K_{1} D^{-1}\right]^{1 / 2}\left[\left(I+K_{1} D\right) \phi-K_{1} C x\right] .
\end{aligned}
$$

Since $R$ is positive definite and $\left(\phi-K_{1} y\right)^{\mathrm{T}}\left(\phi-K_{2} y\right) \leqslant 0$ for all $\phi \in \Phi_{\mathrm{c}}$, it follows that $\Delta V(x)$ is negative definite. Hence $V(x)$ is a Lyapunov function for the negative feedback interconnection of $G(z)$ and $\phi$.

\section{Remark 5.1}

As in the continuous-time case, ${ }^{1}$ considerable simplification can be achieved in (39) by setting $D=0$ which corresponds to a strictly proper $G(z)$. In this case, (39) becomes

$$
\begin{aligned}
P= & \left(A-B K_{1} C\right)^{\mathrm{T}} P\left(A-B K_{1} C\right)+\left[\left(K_{2}-K_{1}\right) C-B^{\mathrm{T}} P\left(A-B K_{1} C\right)\right]^{\mathrm{T}}\left(2 I-B^{\mathrm{T}} P B\right)^{-1} \\
& \times\left[\left(K_{2}-K_{1}\right) C-B^{\mathrm{T}} P\left(A-B K_{1} C\right)\right]+R
\end{aligned}
$$

or, equivalently, using the matrix inversion lemma and grouping terms,

$$
\begin{aligned}
P= & A^{\mathrm{T}} P A+\left[\left(K_{1}+K_{2}\right) C-B^{\mathrm{T}} P A\right]^{\mathrm{T}}\left(2 I-B^{\mathrm{T}} P B\right)^{-1}\left[\left(K_{1}+K_{2}\right) C-B^{\mathrm{T}} P A\right] \\
& -C^{\mathrm{T}}\left(K_{2}^{\mathrm{T}} K_{1}+K_{1}^{\mathrm{T}} K_{2}\right) C+R .
\end{aligned}
$$

As in Reference 1, note that if $K_{2}^{\mathrm{T}} K_{1}+K_{1}^{\mathrm{T}} K_{2} \leqslant 0$, then it follows from (45) that a necessary condition for absolute stability of the negative feedback interconnection of $G(z)$ and $\phi$ is that $A$ be Hurwitz.

Next, as in Sections 3 and 4, we specialize the results of Theorem 5.1 to robust stability of 
a linear time-invariant plant with a linear time-varying uncertainty. First, define

$$
\mathcal{F}_{\mathrm{c}} \triangleq\left\{F: \mathbb{N} \rightarrow \mathbb{R}^{m \times l}:\left[F(k)-K_{1}\right]^{\mathrm{T}}\left[F(k)-K_{2}\right] \leqslant 0, k \in \mathbb{N}\right\}
$$

and consider the system

$$
x(k+1)=(A+\Delta A(k)) x(k),
$$

where $\Delta A(\cdot) \in \mathcal{U}_{\mathrm{c}}$ and the uncertainty set $\mathcal{U}_{\mathrm{c}}$ is defined by

$$
\mathcal{U}_{\mathrm{c}} \triangleq\left\{\Delta A(\cdot): \Delta A(k)=-B F(k)(I+D F(k))^{-1} C, k \in \mathbb{N} \text {, where } F(\cdot) \in \mathcal{F}_{\mathrm{c}}\right\} .
$$

Then it follows from Theorem 5.1, with $\phi(y, k)=F(k) y=F(k) y=F(k)(I+D F(k))^{-1} C x$, that the zero solution to (46) is asymptotically stable for all $\Delta A(\cdot) \in \mathcal{U}_{\mathrm{c}}$. Finally, as in the continuous-time case ${ }^{1}$, if $K_{1}=-I$ and $K_{2}=I$, then $\mathcal{U}_{\mathrm{c}}=\mathcal{U}_{\mathrm{br}}$, while if (formally) $K_{1}=0$ and $K_{2}=\infty$, then $\mathcal{u}_{\mathrm{c}}=\mathcal{U}_{\mathrm{br}}$.

\section{THE POPOV CRITERION}

Ever since Popov derived a frequency-domain condition for the absolute stability of continuous-time nonlinear feedback systems ${ }^{1}$ considerable work has been done to derive similar criteria for discrete-time systems. ${ }^{16-30}$ Unlike the continuous-time case, several different criteria have been proposed for the absolute stability of nonlinear discrete-time systems. Specifically, Tsypkin ${ }^{21}$ has given conditions that involve slope-restricted monotonic functions in the nonlinear element. Significant contributions were also made by Szegö, ${ }^{22,23}$ Jury and Lee $\mathrm{e}^{24,25}$ and Pearson and Gibson ${ }^{26}$ who derived less conservative criteria for the case of slope-bounded nonlinearities. Some extensions to monotonic, odd monotonic, and timevarying gain functions in the feedback path using passive operator techniques have also been considered. ${ }^{27}$ However, the results cited above are confined to scalar, SISO systems with a single loop nonlinearity. In this section we construct Lyapunov functions to prove the discretetime Popov criterion as developed in References $22-24$ for a multivariable plant containing an arbitrary number of memoryless time-invarant nonlinearities. Specialization of this result to robust stability with respect to time-invariant plant uncertainty is also considered. To begin we define the set $\Phi_{P}$ characterizing a class of sector-bounded slope-restricted time-invariant memoryless nonlinearities. Let $K \in \mathbb{R}^{m \times m}$ be a given positive-definite matrix, let $\mu_{1}, \ldots, \mu_{m}$ be positive numbers, and define

$$
\begin{gathered}
\Phi_{\mathrm{P}} \triangleq\left\{\phi: \mathbb{R}^{m} \rightarrow \mathbb{R}^{m}: \phi^{\mathrm{T}}(y)\left[K^{-1} \phi(y)-y\right] \leqslant 0, y \in \mathbb{R}^{m},\right. \\
\left.\phi(y)=\left[\phi_{1}\left(y_{1}\right), \phi_{2}\left(y_{2}\right), \ldots, \phi_{\mathrm{m}}\left(y_{m}\right)\right]^{\mathrm{T}} \text { and } 0<\frac{\phi_{i}(y)-\phi_{i}(\hat{y})}{y-\hat{y}}<\mu_{i}, y, \hat{y} \in \mathbb{R}, i=1, \ldots, m\right\} .
\end{gathered}
$$

For convenience in stating the main result we define $\mu \triangleq \operatorname{diag}\left[\mu_{1}, \mu_{2}, \ldots, \mu_{m}\right]$.

\section{Theorem 6.1}

Suppose there exists a nonnegative-definite diagonal matrix $N$ such that $K^{-1}+[I+(z-1) N] G(z)-\frac{1}{2}|z-1|^{2} G^{*}(z) \mu N G(z)$ is strongly positive real, where

$$
G(z) \stackrel{\min }{\sim}\left[\begin{array}{c|c}
A & B \\
\hline C & 0
\end{array}\right] .
$$


Then there exist positive-definite matrices $P$ and $R$ satisfying

$$
\begin{aligned}
& K^{-1}+N C B+\left(K^{-1}+N C B\right)^{\mathrm{T}}-B^{\mathrm{T}} C^{\mathrm{T}} \mu N C B-B^{\mathrm{T}} P B>0, \\
& P= A^{\mathrm{T}} P A+(A-I)^{\mathrm{T}} C^{\mathrm{T}} \mu N C(A-I)+\left(C+B^{\mathrm{T}} C^{\mathrm{T}} \mu N C+N C A-B^{\mathrm{T}} C \mu N C A-N C-B^{\mathrm{T}} P A\right)^{\mathrm{T}} \\
& \times\left[\left(K^{-1}+N C B\right)+\left(K^{-1}+N C B\right)^{\mathrm{T}}-B^{\mathrm{T}} C^{\mathrm{T}} \mu N C B-B^{\mathrm{T}} P B\right]^{-1} \\
& \times\left(C+B^{\mathrm{T}} C^{\mathrm{T}} \mu N C+N C A-B C^{\mathrm{T}} \mu N C A-N C-B^{\mathrm{T}} P A\right)+R .
\end{aligned}
$$

Furthermore, for all $\phi \in \Phi_{\mathrm{P}}$, the function

$$
V(x)=x^{\mathrm{T}} P x+2 \sum_{i=1}^{m} \int_{0}^{y_{i}(k)} \phi_{i}(\sigma) N_{i} \mathrm{~d} \sigma
$$

is a Lyapunov function for the negative feedback interconnection of $G(z)$ and $\phi$. Consequently, the negative feedback interconnection of $G(z)$ and $\phi$ is asymptotically stable for all $\phi \in \Phi_{\mathrm{P}}$.

Proof. First note that the negative feedback interconnection of $G(z)$ and $\phi(\cdot)$ has the statespace representation

$$
\begin{gathered}
x(k+1)=A x(k)-B \phi(y(k)), \\
y(k)=C x(k) .
\end{gathered}
$$

Next, since $(z-1) G(z)$ has a minimal realization

$$
(z-1) G(z) \stackrel{\min }{\sim}\left[\begin{array}{c|c}
A & B \\
\hline C(A-I) & C B
\end{array}\right],
$$

it follows from Lemma 4.1 and Lemma 4.2 that if $K^{-1}+[I+(z-1) N]$ $G(z)-\frac{1}{2}|z-1|^{2} G^{*}(z) \mu N G(z)$ is strongly positive real, then there exist $P, L, W$ with $P$ positive definite and $W^{\mathrm{T}} W>0$ satisfying

$$
\begin{gathered}
P=A^{\mathrm{T}} P A+L^{\mathrm{T}} L+(A-I)^{\mathrm{T}} C^{\mathrm{T}} \mu N C(A-I), \\
0=B^{\mathrm{T}} P A-C+B^{\mathrm{T}} C^{\mathrm{T}} \mu N C A-B^{\mathrm{T}} C^{\mathrm{T}} \mu N C-N C A+N C+W^{\mathrm{T}} L, \\
0=\left(K^{-1}+N C B\right)+\left(K^{-1}+N C B\right)^{\mathrm{T}}-B^{\mathrm{T}} C^{\mathrm{T}} \mu N C B-B^{\mathrm{T}} P B-W^{\mathrm{T}} W .
\end{gathered}
$$

Next, with $W^{\mathrm{T}} W>0$, it follows that the three equations (52)-(54) collapse to (48). Hence, since $K^{-1}+[I+(z-1) N] G(z)-\frac{1}{2}|z-1|^{2} G^{*}(z) \mu N G(z)$ is strongly positive real, there exist positive-definite matrices $P$ and $R$ such that (47) and (48) are satisfied. Next, for $\phi \in \Phi_{\mathrm{P}}$ define the Lyapunov candidate

$$
V(x)=x^{\mathrm{T}} P x+2 \sum_{i=1}^{m} \int_{0}^{y_{i}(k)} \phi_{i}(\sigma) N_{i} \mathrm{~d} \sigma .
$$

Note that, since $P$ is positive definite and $\phi \in \Phi_{\mathrm{P}}, V(x)$ is positive for all nonzero $x$. Then, the corresponding Lyapunov difference is given by

$$
\Delta V(x)=x^{\mathrm{T}}\left(A^{\mathrm{T}} P A-P\right) x-x^{\mathrm{T}} A^{\mathrm{T}} P B \phi-\phi^{\mathrm{T}} B^{\mathrm{T}} P A x+\phi^{\mathrm{T}} B^{\mathrm{T}} P B \phi+2 \sum_{i=1}^{m} \int_{y_{i}(k)}^{y_{i}(k+1)} \phi_{i}(\sigma) N_{i} \mathrm{~d} \sigma .
$$

Next, using the fact that

$$
0 \leqslant \frac{\phi(y)-\phi(\hat{y})}{y-\hat{y}} \leqslant \mu_{i}
$$


it follows from the mean value theorem that the last term in (55) is bounded from above by

$2 \sum_{i=1}^{m} \int_{y_{i}(k)}^{y_{i}(k+1)} \phi_{i}(\sigma) N_{i} \mathrm{~d} \sigma \leqslant \sum_{i=1}^{m}$

$$
\times\left\{2 \phi_{i}\left(y_{i}(k)\right) N_{i}\left[y_{i}(k+1)-y_{i}(k)\right]+\mu_{i} N_{i}\left[y_{i}(k+1)-y_{i}(k)\right]^{2}\right\}
$$

or, equivalently,

$$
\begin{aligned}
2 \sum_{i=1}^{m} \int_{y_{1}(k)}^{y_{i}(k+1)} \phi_{i}(\sigma) N_{i} \mathrm{~d} \sigma \leqslant & 2 \phi^{\mathrm{T}}(y(k)) N[y(k+1)-y(k)] \\
& +[y(k+1)-y(k)]^{\mathrm{T}} \mu N[y(k+1)-y(k)] .
\end{aligned}
$$

Next, since $y(k+1)=C x(k+1)=C A x(k)-C B \phi(y(k))$ and $y(k+1)-y(k)=C(A-I) x(k)$ - $C B \phi(y(k))$, using (48), (55) becomes

$$
\begin{aligned}
\Delta V(x) \leqslant & -x^{\mathrm{T}} R x-x^{\mathrm{T}}(A-I)^{\mathrm{T}} C^{\mathrm{T}} \mu N C(A-I) x-x^{\mathrm{T}} Q x \\
& +\phi^{\mathrm{T}} N C(A-I) x+x(A-I)^{\mathrm{T}} C^{\mathrm{T}} N \phi-\phi^{\mathrm{T}} N C B \phi-\phi^{\mathrm{T}} B^{\mathrm{T}} C^{\mathrm{T}} N \phi \\
& +x^{\mathrm{T}}(A-I)^{\mathrm{T}} C^{\mathrm{T}} \mu N C(A-I) x-x^{\mathrm{T}}(A-I)^{\mathrm{T}} C \mu N C B \phi \\
& -\phi^{\mathrm{T}} B^{\mathrm{T}} \mu N C(A-I) x+\phi^{\mathrm{T}} B^{\mathrm{T}} C^{\mathrm{T}} \mu N C B \phi-x^{\mathrm{T}} A^{\mathrm{T}} P B \phi \\
& -\phi^{\mathrm{T}} B^{\mathrm{T}} P A x+\phi^{\mathrm{T}} B^{\mathrm{T}} P B \phi,
\end{aligned}
$$

where

$$
\begin{aligned}
Q \triangleq & \left(C+B^{\mathrm{T}} C^{\mathrm{T}} \mu N C+N C A-B^{\mathrm{T}} C^{\mathrm{T}} \mu N C A-N C-B^{\mathrm{T}} P A\right)^{\mathrm{T}} \\
& \times\left[\left(K^{-1}+N C B\right)+\left(K^{-1}+N C B\right)^{\mathrm{T}}-B^{\mathrm{T}} C^{\mathrm{T}} \mu N C B-B^{\mathrm{T}} P B\right]^{-1} \\
& \times\left(C+B^{\mathrm{T}} C^{\mathrm{T}} \mu N C+N C A-B^{\mathrm{T}} C^{\mathrm{T}} \mu N C A-N C-B^{\mathrm{T}} P A\right) .
\end{aligned}
$$

Adding and subtracting $2 \phi^{\mathrm{T}} C x$ and $2 \phi^{\mathrm{T}} K^{-1} \phi$ to and from (56) yields

$$
\begin{aligned}
\Delta V(x) \leqslant & -x^{\mathrm{T}} R x-x^{\mathrm{T}} Q x+\phi^{\mathrm{T}}\left[C+B^{\mathrm{T}} C^{\mathrm{T}} \mu N C(I-A)+N C(A-I)-B^{\mathrm{T}} P A\right] x \\
& +x^{\mathrm{T}}\left[C+B^{\mathrm{T}} C^{\mathrm{T}} \mu N C(I-A)+N C(A-I)-B^{\mathrm{T}} P A\right]^{\mathrm{T}} \phi \\
& -\phi^{\mathrm{T}}\left[\left(K^{-1}+N B C\right)+\left(K^{-1}+N C B\right)^{\mathrm{T}}-B^{\mathrm{T}} C^{\mathrm{T}} \mu N C B-B^{\mathrm{T}} P B\right] \phi \\
& +2 \phi^{\mathrm{T}}\left[K^{-1} \phi-C x\right]
\end{aligned}
$$

or, equivalently,

$$
\Delta V(x) \leqslant-x^{\mathrm{T}} R x-z^{\mathrm{T}} z+2 \phi^{\mathrm{T}}\left[K^{-1} \phi-y\right]
$$

where

$$
\begin{aligned}
z \triangleq & {\left[\left(K^{-1}+N C B\right)+\left(K^{-1}+N C B\right)^{\mathrm{T}}-B^{\mathrm{T}} C^{\mathrm{T}} \mu N C B-B^{\mathrm{T}} P B\right]^{-1 / 2} } \\
& \times\left(C+B^{\mathrm{T}} C^{\mathrm{T}} \mu N C(I-A)+N C(A-I)-B^{\mathrm{T}} P A\right) x \\
& -\left[\left(K^{-1}+N C B\right)+\left(K^{-1}+N C B\right)^{\mathrm{T}}-B^{\mathrm{T}} C^{\mathrm{T}} \mu N C B-B^{\mathrm{T}} P B\right]^{1 / 2} \phi .
\end{aligned}
$$

Since $R$ is positive definite and $\phi^{\mathrm{T}}\left[K^{-1} \phi-y\right] \leqslant 0$, it follows that $\Delta V(x)$ is negative definite. Hence $V(x)$ is a Lyapunov function for the negative feedback interconnection of $G(z)$ and $\phi$.

\section{Remark 6.1}

Theorem 6.1 presents a generalization of the discrete-time Popov criterion ${ }^{22-24}$ to the case of a multivariable plant containing an arbitrary number of memoryless time-invariant 
nonlinearities. It should also be noted that a similar proof using (52)-(54) rather than (48) can also be constructed. In this case the condition $K^{-1}+[I+(z-1) N] G(z)-\frac{1}{2}|z-1|^{2} G^{*}(z) \mu N G(z)$ can be relaxed from strongly positive real to positive real. However, we present the form of (48) since it provides an algebraic formulation in terms of a matrix Riccati equation which can be used to synthesize robust feedback controllers in the spirit of References 31 and 32 .

Now we specialize the results of Theorem 6.1 to linear parameter uncertainty, in which case $V(x)$ becomes quadratic. Define the set

$$
\mathcal{F}_{\mathrm{P}} \triangleq\left\{F \in \mathbb{R}^{m \times m}: F=\operatorname{diag}\left[F_{1}, \ldots, F_{m}\right], 0 \leqslant F_{i} \leqslant \mu_{i}, i=1, \ldots, m\right\}
$$

of constant diagonal matrices $F$ where $\mu_{1}, \ldots, \mu_{m}$ are positive constants. Next consider the system

$$
x(k+1)=(A+\Delta A) x(k),
$$

where the constant matrix $\Delta A$ satisfies $\Delta A \in \mathcal{U}_{\mathrm{P}}$, where

$$
\mathfrak{F}_{\mathrm{P}} \triangleq\left\{\Delta A: \Delta A=-B F C \text {, where } F \in \mathcal{F}_{\mathrm{P}}\right\} .
$$

It now follows from Theorem 6.1 by setting $\phi(y)=F y=F C x$ that $A+\Delta A$ is asymptotically stable for all $\Delta A \in \mathcal{U}_{\mathrm{P}}$.

As in the continuous-time case, ${ }^{1}$ the results of this section differ from the previous sections in that the elements of the set $\mathcal{U}_{\mathrm{P}}$ are constant rather than time-varying. Once again this is due to the form of the Lyapunov function that establishes robust stability, i.e.,

$$
V(x)=x^{\mathrm{T}} P x+2 \sum_{i=1}^{m} \int_{0}^{y_{i}(k)} F_{i} \sigma \mathrm{d} \sigma, y_{i}=C_{i} x,
$$

or, equivalently,

$$
V(x)=x^{\mathrm{T}} P x+x^{\mathrm{T}} C^{\mathrm{T}} F C x .
$$

This quadratic Lyapunov function is parameter-dependent. ${ }^{31,32}$ The form of $V(x)$ is critical since the presence of $F$ restricts the allowable time-varying uncertain parameters thus reducing conservatism for the real-parameter uncertainty problem. ${ }^{33}$

\section{CONCLUSION}

In this two-part paper we constructed Lyapunov functions for the small gain, positivity, circle, and Popov theorems for both continuous-time and discrete-time settings for interconnections involving a linear time-invariant transfer function and a memoryless nonlinearity. Each result was then specialized to the problem of robust stability involving linear uncertainty. As in the continuous-time case, it can be shown that the results of this paper also apply to the problem of robust $\mathrm{H}_{2}$ performance. To see this, consider the discrete-time asymptotically stable nominal linear system

$$
x(k+1)=A x(k)
$$

with quadratic Lyapunov function

$$
V(x)=x^{\mathrm{T}} P x,
$$

where the positive-definite matrix $P$ is given by

$$
P=A^{\mathrm{T}} P A+R,
$$


where $R$ is positive definite. Next, to address additive disturbances for a system of the form

$$
x(k+1)=A x(k)+w(k),
$$

consider the dual equation

$$
Q=A Q A^{\mathrm{T}}+V,
$$

where $V$ is interpreted as the covariance of the disturbance $w(\cdot)$. As in the continuous-time case, the matrix $Q$ can be viewed as a discrete-time controllability Gramian or covariance matrix with associated quadratic $\mathrm{H}_{2}$ performance measure $J=\operatorname{tr} Q R=\operatorname{tr} P V$.

Now suppose $A$ is uncertain so that

$$
x(k+1)=(A+\Delta A) x(k), \Delta A \in \mathcal{U},
$$

where $U$ is a set of perturbations. To determine whether $A+\Delta A$ remains stable, we replace (61) by

$$
P=A^{\mathrm{T}} P A+\Omega(P)+R,
$$

where $\Omega(\cdot)$ satisfies

$$
A^{\mathrm{T}} P \Delta A+\Delta A^{\mathrm{T}} P A+\Delta A^{\mathrm{T}} P \Delta A \leqslant \Omega(P), \Delta A \in \mathcal{U},
$$

and for all positive-definite $P$. Rewriting (65) as

$$
P=(A+\Delta A)^{\mathrm{T}} P(A+\Delta A)+\Omega(P)-\left(A^{\mathrm{T}} P \Delta A+\Delta A^{\mathrm{T}} P A+\Delta A^{\mathrm{T}} P \Delta A\right)+R
$$

shows that $A+\Delta A$ is stable and

$$
P_{\Delta A} \leqslant P, \Delta A \in \mathcal{U},
$$

where $P_{\Delta A}$ satisfies

$$
P_{\Delta A}=(A+\Delta A)^{\mathrm{T}} P_{\Delta A}(A+\Delta A)+R .
$$

Thus, as in the continuous-time $\Omega$-bound framework, ${ }^{1} \operatorname{tr} P V$ provides a worst case bound for the actual $\mathrm{H}_{2}$ performance tr $P_{\triangle A} V$.

Although the $\Omega$-bound framework discussed above applies to problems in which $A$ is perturbed by an uncertainty $\Delta A$, a reinterpretation of this framework yields the well-known system-theoretic criteria discussed in this paper. Specifically, assuming $\Delta A$ to be of the form,

$$
\Delta A=B F C, \sigma_{\max }(F) \leqslant 1,
$$

where $F$ is an uncertain real matrix and $B$ and $C$ are known matrices denoting the structure of the uncertainty, it follows from Corollary 3.1 that

$$
\Omega(P)=C^{\mathrm{T}} C+A^{\mathrm{T}} P B\left(I-B^{\mathrm{T}} P B\right)^{-1} B^{\mathrm{T}} P A
$$

satisfies (65) with $\mathcal{U}=\mathcal{U}_{\mathrm{br}}$ (for the case $D=0$ ). Similar constructions apply for the positivity and circle theorems.

As in the continuous-time case, similar remarks on conservatism apply when $\Delta A$ is constant since, using this framework, stability is guaranteed even if $\Delta A$ is a function of $k$. Once again, however, to address the constant real parameter uncertainty problem one needs a more refined discrete-time $\Omega$-bound. An immediate application of this framework is a reinterpretation of the classical discrete-time Popov criterion as a parameter-dependent Lyapunov function.

To demonstrate this in discrete-time, consider the Lyapunov function $V(x, \Delta A)=$ $x^{\mathrm{T}}\left(P+P_{0}(\Delta A)\right) x$ where the (parameter-independent) matrix $P$ satisfies

$$
P=A^{\mathrm{T}} P A+\Omega_{0}(P)+R
$$


and $\Omega_{0}(P)$ satisfies

$$
A^{\mathrm{T}} P \Delta A+\Delta A^{\mathrm{T}} P A+\Delta A^{\mathrm{T}} P \Delta A \leqslant \Omega_{0}(P)-\left[(A+\Delta A)^{\mathrm{T}} P_{0}(\Delta A)(A+\Delta A)-P_{0}(\Delta A)\right],
$$

for all $\Delta A \in \mathcal{U}$. Letting

$$
\Omega(P, \Delta A) \triangleq \Omega_{0}(P)-\left[(A+\Delta A)^{\mathrm{T}} P_{0}(\Delta A)(A+\Delta A)-P_{0}(\Delta A)\right]
$$

it follows from (71) that

$$
\begin{aligned}
P+P_{0}(\Delta A)= & (A+\Delta A)^{\mathrm{T}}\left(P+P_{0}(\Delta A)\right)(A+\Delta A)+\Omega(P, \Delta A) \\
& -\left(A^{\mathrm{T}} P \Delta A+\Delta A^{\mathrm{T}} P+\Delta A^{\mathrm{T}} P \Delta A\right)+R,
\end{aligned}
$$

which implies that $A+\Delta A$ is stable for all $\Delta A \in \mathcal{U}$. One can now show that the discrete-time Popov criterion presented in Theorem 6.1 fits the above framework. Furthermore subtracting (68) from (73) yields $P_{\Delta A} \leqslant P+P_{0}(\Delta A)$ and hence $\operatorname{tr}\left[\left(P+\bar{P}_{0}\right) V\right]$ provides a worst case bound for the actual $\mathrm{H}_{2}$ performance $\operatorname{tr} P_{\Delta A} V$ where $\bar{P}_{0} \leqslant P_{0}(\Delta A)$ for all $\Delta A \in \mathcal{U}$.

The underlying intention of this two-part paper is the unification and extension of the absolute stability criteria for linear time-invariant dynamic systems with loop nonlinearities which were developed in the early 1960 s and the modern-day robust stability and $\mathrm{H}_{2}$ performance problems for state-space systems using fixed and parameter-dependent Lyapunov functions. This exposition thus demonstrates that these results are all derivable from the same principles and are part of the same mathematical framework.

\section{ACKNOWLEDGEMENTS}

The authors would like to thank Professors M. G. Safonov and D. D. Siljak for their valuable discussions.

This research was supported in part by the Air Force Office of Scientific Research under Grant F49620-92-J-0127, the National Science Foundation under Research Initiation Grant ECS-9109558, and the National Aeronautics and Space Administration under Contract NAS8-38575.

\section{REFERENCES}

1. Haddad, W. M., and D. S. Bernstein, 'Explicit construction of quadratic Lyapunov functions for the small gain, positivity, circle, and Popov theorems and their application to robust stability. Part I: Continuous-time theory', Int. J. Robust and Nonlinear Control, 3, 313-339 (1993).

2. Vaidyanathan, P. P., 'The discrete-time bounded-real lemma in digital filtering', IEEE Trans. Circ. Sys., CAS-32, 918-924 (1985).

3. Agathoklis, P., E. I. Jury and M. Mansour, 'The discrete-time strictly bounded-real lemma and the computation of positive definite solutions to the 2-D Lyapunov equation', IEEE Trans. Circ Sys., CAS-36, 830-837 (1989).

4. Hitz, L., and B. D. O. Anderson, 'Discrete positive-real functions and their application to system stability', Proc. IEE, 118, 153-155 (1969).

5. Siljak, D. D., 'Algebraic criteria for positive realness relative to the unit circle', J. Franklin Inst., 295, 469-476 (1973).

6. Cains, P. E., Linear Stochastic Systems, Wiley, New York, 1989.

7. Chu, C. C., 'On discrete inner-outer and spectral factorizations', Proc. Amer. Contr. Conf., 1699-1700, Atlanta, GA (1988).

8. Molinari, B. P., 'The stabilizing solution of the discrete algebraic Riccati equation', IEEE Trans. Autom. Contr., AC-20, 396-399 (1975).

9. Kalman, R. E., and J. E. Bertram, 'Control system analysis and design via the 'second method' of Lyapunov II discrete-time systems', Trans. ASME, 82, 394-400, (1960).

10. Haddad, W. M., H.-H. Huang and D. S. Bernstein, 'Robust stability and performance via fixed-order dynamic compensation: the discrete-time case', IEEE Trans. Autom. Contr., to appear.

11. Tsypkin, Y. Z., 'Fundamentals of the theory of nonlinear pulse control systems', Proc. 2nd IFAC Cong., Butterworths, London, 1964. 
12. Tsypkin, Y. Z., 'Frequency criteria for the absolute stability of nonlinear sampled-data systems', Aviom. $i$ Telemek., 25, 281-289 (1964).

13. Sandberg, I. W., 'On the boundedness of solutions of nonlinear integral equations', Bell Sys. Tech. J., 44, 439-453 (1965).

14. Wu, S. H., 'A circle stability criteria for a class of discrete system', IEEE Trans. Autom. Contr., AC-12, 114-115 (1967).

15. Lee, T.-T., and S.-H. Lee, 'Gain and phase margins for discrete-time systems', Int. J. Conirol, 44, 1415-1426 (1986).

16. Tsypkin, Y. Z., 'On the stability in the large of nonlinear sampled-data systems', Dokl. Akad. Nauk., 145, 52-55 (1962).

17. Kalman R. E., and G. Szegö, 'Sur la stabilité d'un système d'équations aux différences finies', CR Acad. Sci. Paris, 257, 388-390 (1963).

18. Kodama, S., 'Stability of nonlinear sampled-data control systems', IEEE Trans. Autom. Contr., AC-7, 15-23 (1962).

19. Kodama, S., 'Stability of a class of discrete control systems containing a nonlinear gain element', IEEE Trans. Autom. Contr., AC-7, 102-109 (1962).

20. Pearson, Jr., J. B., 'A note on the existence of Liapunov functions for a class of discrete-time systems', IEEE Trans. Auto. Contr., AC-8, 262-263 (1963).

21. Tsypkin, Y. Z., 'A criterion for absolute stability of automatic pulse systems with monotonic characteristics of the nonlinear element', Sov. Phys.-Doklady, 9, 263-266 (1964).

22. Szegö, G., 'On the absolute stability of sampled-data control systems', Proc. Nat. Acad. Sci., 49, 558-560 (1963).

23. Szegö, G., and J. B. Pearson, Jr., 'On the absolute stability of sampled-data systems: the indirect control case', IEEE Trans. Autom. Contr., AC-9, 160-163 (1964).

24. Jury, E. I., and B. W. Lee, 'On the stability of a certain class of nonlinear sampled-data systems', IEEE Trans. Autom. Contr., AC-9, 51-61 (1964).

25. Jury, E. I., and B. W. Lee, 'On the absolute stability of nonlinear sampled-data systems', IEEE Trans. Autom. Contr., AC-9, 551-554 (1964).

26. Pearson, Jr., J. B., and J. E. Gibson, 'On the asymptotic stability of a class of saturating sampled-data systems', IEEE Trans. Appl. Ind., 83, 81-86 (1964).

27. Narendra, K. S., and Y.-S. Cho, 'Stability Analysis of nonlinear and time-varying discrete-systems', SIAM J. Contr., 6, 625-646 (1968).

28. Shortle, Jr., G. E., and F. J. Alexandro, Jr., 'An extended frequency-domain stability criterion for a class of sampled-data systems', IEEE Trans. Autom. Contr., AC-17, 365-368 (1972).

29. O'Shea, R. P., and M. I. Younis, 'A frequency-time domain stability criterion for sampled-data systems', IEEE Trans. Autom. Contr., AC-12, 719-724 (1967).

30. Siljak, D. D., and C. K. Sun, 'Exponential absolute stability of discrete-systems', ZAMM, 51, 271-275 (1971).

31. Haddad, W. M., and D. S. Bernstein, 'Parameter-dependent Lyapunov functions, constant real parameter uncertainty and the Popov criterion in robust analysis and synthesis', submitted to IEEE Trans. Auto. Contr.

32. Haddad, W. M., and D. S. Bernstein, 'Parameter-dependent Lyapunov functions and the discrete-time Popov criterion for robust analysis', Automatica, to appear.

33. Bernstein, D. S., W. M. Haddad and D. C. Hyland, 'Small gain versus positive real modelling of real parameter uncertainty', AIAA J. Guid. Contr. Dyn., 15, 538-540 (1992). 